\title{
Inovação: gasto ou investimento?
}

\author{
Déborah Mara Siade Barbosa \\ Doutorado em andamento em Administração pela Universidade Federal de Minas \\ Gerais - UFMG \\ Avenida Antônio Carlos, 6627. Pampulha. Belo Horizonte/MG. CEP: 31270-901 \\ E-mail: deborahsiade@yahoo.com.br
}

Nayara Silva de Noronha

Doutorado em andamento em Administração pela Fundação Getúlio Vargas - FGV

Rua Itapeva, 432. Bela Vista. São Paulo/SP. CEP: 01313-902

E-mail:nayara.noronha@gmail.com

Thaís Alves dos Santos

Mestrado em Administração pela Universidade Federal de Lavras - UFLA

Professora do Centro Mineiro do Ensino Superior

Rua Projetada, s/no. Arnaldo. Campo Belo/MG. CEP: 37270-970

E-mail: thatha226@yahoo.com.br

Mário Sérgio de Almeida Doutorado em andamento da Universidade Federal de Lavras - UFLA

Câmpus Universitário. Lavras/MG. CEP: 37200-000

E-mail:mario_s_a@yahoo.com.br

\section{RESUMO}

No atual mercado competitivo a inovação tem sido percebida como fator de vantagem competitiva e muitos investimentos são realizados visando o desenvolvimento de inovações. Entretanto, tais investimentos se configuram em ganhos financeiros para as firmas, ou seja, são, realmente, investimentos ou somente gastos? Para compreender tal questionamento duas hipóteses foram levantadas. A primeira hipótese, $\mathrm{H}_{1}$, é a de que o valor de mercado da firma é influenciado pela inovação. A segunda hipótese, $\mathrm{H}_{2}$, é a de que a capacidade operacional de geração de riqueza da empresa é influenciada pela inovação. Sendo esta uma pesquisa quantitativa, de caráter descritivo, utilizou-se o software SPSS para realizar regressão múltipla dos dados de cinco grandes empresas que divulgaram os dados de intangíveis e goodwill no banco de dados Economática. Os resultados encontrados confirmam as hipótese $\mathrm{H}_{1}$ e $\mathrm{H}_{2}$. Assim, pode-se afirmar que o valor de mercado das empresas é influenciado pela inovação, no entanto, os ativos intangíveis e o goodwill não permitem inferir se a inovação influencia na capacidade operacional de gerar riqueza da empresa.

Palavras-chave: Inovação. Gasto. Investimento, Goodwill. 
Inovação: Gasto ou Investimento?

Déborah Mara Siade Barbosa, Nayara Silva de Noronha, Thaís Alves dos Santos, Mário Sérgio de

Almeida

\title{
Innovation: expense or investment?
}

\begin{abstract}
In today's competitive market, innovation has been perceived as a factor of competitive advantage and many investments are made for the development of innovations. However, such investments are configured in financial gains for firms, ie, they are really only spending or investments? To understand

such questioning two hypotheses were raised. The first hypothesis, $\mathrm{H} 1$, is that the market value of the firm is influenced by innovation. The second hypothesis, $\mathrm{H} 2$, is that the operational capacity of the company's wealth creation is influenced by innovation. Since this quantitative study was descriptive in character, we used SPSS software to perform multiple regression analysis of data from five major companies that released the data of intangibles and goodwill in the database Economática. The results show that the hypothese $\mathrm{H}_{1}$ can be accepted and $\mathrm{H}_{2}$ refuted. Thus, one can say that the market value of firms is influenced by innovation, however, intangible assets and goodwill not allow inferring the influence innovation in the operational capacity of the company to generate wealth.
\end{abstract}

Keywords: Innovation. Expenses. Investment. Goodwill.

\section{INTRODUÇÃO}

Diante do contexto corporativo atual é crescente a necessidade de as empresas lançarem inovações em caráter contínuo, a fim de se manterem competitivas. Dados da Pesquisa de Inovação Tecnológica - PINTEC (IBGE) demonstram a necessidade de as empresas desenvolverem competências que as mantenham competitivas, bem como possuírem flexibilidade organizacional para atenderem, de maneira ágil e constante, às necessidades prementes do mercado. A inovação aparece como ator neste processo competitivo, visto ser considerada geradora de diferenciação e, portanto, fonte de geração de valor para os negócios das firmas.

Para que a firma atenda tais requisitos é preciso investimento, isto é, é necessário que dispêndios sejam realizados para que haja inovações constantes. Resta saber se estes implicam, de fato, em geração de valor para as firmas. A relevância de 
medir a inovação pode ter usos diversos. Entretanto, o foco principal está na possibilidade de compreender de maneira mais acurada a relação entre inovação e crescimento econômico (OECD, 2005).

Em se tratando de desenvolvimento econômico, as firmas são a força motriz desse processo. Deste modo, compreender o impacto das inovações nas empresas é de fundamental importância. Optou-se por utilizar ativos intangíveis e goodwill como indicadores de investimento em inovação nas empresas. Esta escolha está apoiada em resultados de estudos nacionais (TEH; KAYO; KIMURA, 2008; TIRONI; CRUZ, 2008; COLAUTO et al., 2009; CRISÓSTOMO, 2009) e internacionais (ROGERS, 1998; LEV, 2001; DARROCH; MCNAUGHTON, 2002; EDVINSSON et al., 2004; NARVEKAR; KARUNA, 2006; MUDAMBI, 2008).

Ademais, a terceira edição do Manual de Oslo que versa sobre a coleta e interpretação de dados sobre inovação, aponta que a compra de ativos intangíveis com potencial de rentabilidade em longo prazo está relacionada com a inovação (OECD, 2005). Destarte, considerando a vigência da era do conhecimento e que quanto maior o grau de novidade maior a necessidade de intangíveis na atividade inovadora (TIRONI; CRUZ, 2008), acredita-se que é possível mensurar inovação utilizando o investimento em ativos intangíveis e em goodwill.

Considerando o cenário competitivo e a necessidade de inovação para se manter no mercado, julga-se essencial mensurar se os investimentos em inovação geram benefícios concretos para as firmas, ou seja, se a inovação impacta no crescimento do valor de mercado da firma e em sua capacidade de geração de riqueza. Decorrente de tal problematização, a questão que baliza o presente estudo é: os investimentos realizados em inovação se configuram em ganhos financeiros para as firmas, isto é, são de fato investimentos ou gastos?

Para o desenvolvimento da pesquisa balizada por essa questão, foram propostas duas hipóteses. A primeira hipótese, doravante denominada $\mathrm{H}_{1}$, é a de que o valor de mercado da firma é explicado pela inovação. A segunda hipótese, $\mathrm{H}_{2}$, é a de que a 
Inovação: Gasto ou Investimento?

Déborah Mara Siade Barbosa, Nayara Silva de Noronha, Thaís Alves dos Santos, Mário Sérgio de

Almeida

capacidade operacional de geração de riqueza da empresa é influenciada pela inovação.

Esta pesquisa possui como objetivo geral identificar a relação entre inovação e ganhos financeiros para a empresa. Visando atingir o objetivo geral, tem-se como objetivos específicos (i) verificar se a inovação determina retornos financeiros para a empresa e (ii) averiguar se empresas com maiores retornos financeiros são as que mais inovam.

Este artigo é composto por cinco sessões. Além desta introdução, foi realizada revisão de literatura que contempla a dinâmica Schumpeteriana da concorrência e, consequentemente, a necessidade de inovar, bem como a vantagem que as grandes empresas têm sobre as menores para tal e, por último, as possibilidades de mensuração da inovação. $\mathrm{Na}$ terceira sessão são apresentados os processos metodológicos e, em seguida, os resultados encontrados. Na última sessão são tecidas algumas considerações finais e sugestões de pesquisas futuras.

\section{ANTECEDENTES TEÓRICOS}

Aborda-se aqui os conceitos de inovação e concorrência Schumpeteriana tendo em vista o caráter dinâmico do processo inovativo, bem como a relação existente entre as grandes corporações e a inovação e, em seguida, a possibilidade de utilização de indicadores para a mensuração da inovação nas empresas, neste estudo, considerando-se os ativos intangíveis e o goodwill.

\subsection{Inovação e a concorrência Schumpeteriana}

Para Schumpeter (1982), a introdução e a ampliação de inovações tecnológicas e organizacionais nas empresas constituem fatores essenciais para as transformações na esfera econômica e seu desenvolvimento em longo prazo. Para este autor, o sistema econômico apresenta somente mudanças contínuas que não levam ao rompimento do estado de equilíbrio do sistema econômico. Nesse contexto, a 
introdução de uma inovação, desde que seja absorvida pelo mercado, implica em dinamismo para a economia.

O desenvolvimento econômico é visto por Schumpeter como dinâmico e em transformação. Além disso, o processo de inovação é descontínuo, visto que uma inovação é sempre seguida por outra (Schumpeter, 1982). Tal processo é fruto da concorrência inter-firmas, pois, nesse sentido, qualquer inovação é entendida como resultado da busca constante de lucros extraordinários, mediante a obtenção de vantagens competitivas entre as empresas que procuram se diferenciar umas das outras nas mais variadas dimensões do processo competitivo (KUPFER; HASENCLEVER, 2002).

Para Schumpeter (1982), o processo de mudanças tecnológicas que revoluciona incessantemente a estrutura econômica, criando elementos novos e destruindo os antigos, é o processo de destruição criadora. A destruição criadora é o impulso fundamental que aciona e mantém a marcha capitalista, constantemente criando novos produtos, novos métodos de produção, novos mercados e, implacavelmente, sobrepondo-se aos antigos métodos menos eficientes e mais caros (SCHUMPETER, 1982). Esta dinâmica é produto do processo concorrencial entre as grandes empresas monopolistas e oligopolistas. Neste processo as grandes empresas se alternam no ganho de lucros extraordinários, por meio da inserção de inovações (KUPFER; HASENCLEVER, 2002).

A concorrência Schumpeteriana, segundo Kupfer e Hasenclever (2002), caracteriza-se pela busca permanente de diferenciação entre as empresas, por meio de estratégias deliberadas, tendo em vista a obtenção de vantagens competitivas que proporcionem lucros de monopolistas. Cabe ressaltar que a concorrência não se dá somente considerando o critério de preços, mas também por diferenciação do produto e, especialmente, por inovações que envolvem toda e qualquer criação de novos espaços econômicos.

Além disso, os lucros advindos da inovação contribuem para acirrar a competição capitalista, atraindo para o mercado o que Schumpeter denomina de 
"imitadores". Analisando este processo economicamente, em um primeiro momento, as taxas de investimentos, de nível de emprego e de crescimento da economia se elevam e, em seguida, a oportunidade de realização de altos lucros na área associada à inovação é reduzida, haja vista 0 aumento de oferta que ocorre nessa ocasião (SHIKIDA; BACHA, 1996).

Neste contexto concorrencial, Schumpeter (1982) destaca que as inovações tendem a se concentrar em alguns setores da economia, especificamente, nos mais fortes e o processo de difusão dessas inovações se dá de forma desigual. Ademais, as firmas com maior probabilidade de inovar procurarão manter-se na dianteira do progresso técnico, introduzindo inovações, de forma sequenciada, a fim de não se tornarem vítimas desse processo de inovação (MOREIRA, 1989).

\subsection{As grandes corporações e a inovação}

A relação entre a concorrência e a inovação é levantada por Penrose (1958) ao apresentar a importância de a empresa se manter atualizada para competir com as demais organizações. A autora também assevera que a lucratividade continuada da firma está associada às possibilidades de inovação.

São as grandes empresas que, no processo concorrencial, aceleram grandemente a criação de inovação. Penrose (1958) associa o processo Schumpeteriano de destruição criadora ao crescimento da firma. Tal processo não destrói a grande empresa, pelo contrário a impele a tornar-se cada vez mais criativa. Esse afã por criatividade tornou-se predominante no cenário global de competição.

As inovações são consideradas de suma relevância por permitirem às firmas o acesso a novos mercados, o incremento na geração de receitas, a realização de novas parcerias, a aquisição de conhecimentos e o aumento do valor de suas marcas, entre outros. As grandes empresas, em geral, são o centro da inovação, devido à sua capacidade de acompanhar as constantes mudanças, adquirindo vantagem competitiva. Segundo o Instituto Inovação, grande parte das grandes organizações possui áreas inteiras dedicadas à inovação, com laboratórios de pesquisa e 
desenvolvimento (P\&D) que contam com diversos pesquisadores engajados, exclusivamente, no desenvolvimento de inovações. Usualmente, é por meio das grandes firmas que as tecnologias, invenções, produtos, enfim, ideias, chegam ao mercado. Afinal, por serem grandes, elas detém maior poderio de investimentos, maior valor de mercado e, portanto, maior capacidade de geração de inovações.

Tal ideia corrobora a teoria de Penrose (1958), de que a maioria das inovações dos processos produtivos origina-se de firmas industriais e as primeiras firmas a introduzi-las são as que tendem a conseguir vantagem competitiva. O simples fato de terem sido as primeiras a entrarem no mercado já se apresenta como prerrogativa competitiva, no entanto, as inovações, também podem ser protegidas por meio de patentes.

Assim, o processo de crescimento da firma está fortemente ligado à sua capacidade inovadora. As estratégias de diversificação, neste contexto, surgem como forma de expansão das grandes empresas e como busca de novas oportunidades lucrativas em um ambiente competitivo. Na visão de Chandler (apud CARVALHO JUNIOR; RUIZ, 2008), a capacidade das grandes empresas suportarem a diversificação por meio de uma estrutura organizacional e gerencial é o que ocasiona essa vantagem O desempenho da firma, normalmente, é medido pela lucratividade, pela participação no mercado e pelos diferenciais de produtividade setorial (PENROSE, 1958). No entanto, nas próprias palavras da autora "uma firma representa mais do que uma unidade administrativa; trata-se também de um conjunto de recursos produtivos cuja disposição entre diversos usos e através do tempo é determinada por decisões administrativas" (PENROSE, 1958, p. 61). Dentre esses recursos se destacam, principalmente, os recursos intangíveis.

Deste modo, a capacitação tecnológica e o desempenho inovador são apontados como altamente relacionados com o desempenho e a eficiência das firmas. Isso vem ao encontro dos estudos recentes sobre a importância da inovação no cenário econômico, no entanto, a mensuração desse processo é complexa, sobretudo, pela intangibilidade dos ativos que compõem o processo de inovação. 
Inovação: Gasto ou Investimento?

Déborah Mara Siade Barbosa, Nayara Silva de Noronha, Thaís Alves dos Santos, Mário Sérgio de

Almeida

\subsection{A inovação e a intangibilidade de ativos}

O processo de inovação e sua mensuração é um tema controverso. Não existe uma consonância sobre quais variáveis são necessárias para explicar o esforço inovador, a natureza da inter-relação entre estas e qual a mensuração empírica mais adequada. A multidimensionalidade da atividade inovadora e a importância do conhecimento no processo de inovação são elementos difíceis de medir (MATESCO, 1993).

Deste modo, Rios e Pinto (2004) afirmam que saber como mensurar a contribuição da inovação dentro das empresas é um dos maiores problemas desta temática, uma vez que a inovação é apontada como um dos principais atributos do aumento da competitividade entre as empresas. Crisóstomo (2009) alega que a riqueza de uma empresa passa a ser gerada pela inovação.

Ademais, como a inovação envolve investimento relevante, incluindo a aquisição de ativos intangíveis com potencial rentabilidade no longo prazo (OECD, 2005), a mensuração deste torna-se de fundamental importância, no entanto, configura tarefa complexa e de difícil consenso.

A apuração de ativos intangíveis na contabilidade é algo recente, cujo início se deu a partir da Lei $n^{0}$ 11.638/2007 que tornou obrigatória a introdução do intangível no ativo não circulante no balanço patrimonial. A descoberta do valor de ativos intangíveis para as empresas, constituindo uma reserva de benefícios futuros para as organizações, torna este tema relevante e ainda pouco estudado (IUDíCIBUS, 2004).

Ativo intangível é conceituado por ludícibus (2004, p.225) como "um ativo de capital que não tem existência física, cujo valor é limitado pelos direitos e benefícios que antecipadamente sua posse confere ao proprietário". Sendo assim, pode-se afirmar que para o processo de inovação dentro das empresas, os ativos intangíveis desempenham papel importante. Alguns ativos intangíveis, geralmente oriundos de inovação e conhecimento, como marcas, patentes, capital intelectual e direitos autorais 
Inovação: Gasto ou Investimento?

Déborah Mara Siade Barbosa, Nayara Silva de Noronha, Thaís Alves dos Santos, Mário Sérgio de

Almeida

são ativos singulares (PEREZ; FAMÁ, 2006). No entanto, tais ativos nem sempre são facilmente identificáveis e mensuráveis, como afirmam Antunes e Leite (2008), especialmente aqueles relacionados aos recursos humanos e a P\&D. De acordo com Hendricksen e Van Breda (1999), um dos ativos de mais difícil mensuração é o goodwill.

Marion (2011) defende que o goodwill é um tipo de ágio que a firma possui em decorrência de patentes registradas, treinamento e habilidades de seus funcionários, de sua imagem, sua reputação, da marca de seus produtos, direitos exclusivos de comercialização entre outros. Assim, entende-se que o goodwill pode ser um indicador de mensuração de inovação devido à sua capacidade de medir esses ativos que contribuem para o desenvolvimento das inovações dentro da empresa.

Para fins de apuração do retorno financeiro proporcionado pela inovação, optouse por utilizar o EBITDA - Earning Before Interest, Taxes, Depreciation and Amortization, no Brasil denominado como LAJIDA - lucro antes do pagamento de juros, impostos, depreciação e amortização - que, conforme Vasconcelos (2002), é relevante por ser um misto de indicador de desempenho econômico-financeiro e base para avaliação de empresas. Além disso, o EBITDA demonstra a capacidade operacional de geração de caixa de uma empresa (VASCONCELOS, 2002), assim, quanto maior o EBITDA de uma firma maior a capacidade desta render retorno aos investimentos realizados (ASSAF NETO, 2002).

\section{METODOLOGIA}

O presente estudo pode ser classificado como descritivo, haja vista examinar a existência de associações entre variáveis (GIL, 2002). Ademais, de acordo com Medeiros (2008, p. 30) a presente pesquisa pode ser considerada como descritiva, pois objetiva o "estudo, análise, registro e interpretação dos fatos do mundo físico sem a interferência do pesquisador". Seu enfoque é quantitativo por utilizar-se de ferramentas estatísticas para a realização da análise dos dados (RICHARDSON, 1989). 
O método de pesquisa utilizado foi a análise quantitativa que, segundo Vergara (2006), tem o propósito de identificar as relações entre as variáveis e se caracteriza pela objetividade, utilizando técnicas estatísticas para o tratamento dos dados e buscando a generalização dos resultados.

As unidades de análise foram empresas de capital aberto, cujos dados financeiros estão disponíveis ao mercado. Para coleta destes dados, utilizou-se o banco de dados Economática. Os dados coletados se referem ao quarto trimestre de $2010 \mathrm{e}$ aos quatro trimestres de 2011.

Este espaço temporal foi escolhido devido à publicação da Instrução CVM no 457, editada em julho de 2007, em alinhamento com o Comunicado oㅜ 14.259 do Banco Central do Brasil, de março de 2006 em que a CVM determinou que as companhias brasileiras de capital aberto passassem a elaborar as demonstrações financeiras consolidadas com base nas International Accounting Standards Board (IASB) a partir do exercício de 2010 (CVM, 2007). Uma das modificações que atende aos padrões internacionais é a presença obrigatória do goodwill nos demonstrativos financeiros das firmas.

Foram selecionadas as cinco maiores empresas em operação cujos dados relativos aos gastos com intangíveis e goodwill, no período estipulado, estavam disponíveis. As empresas selecionadas foram: Gafisa, Gol, M. Dias Branco, Marisol, Vulcabrás e Wilson Sons pertencentes aos setores de construção, transporte e serviços, alimentos e bebidas, têxtil e transporte serviços, respectivamente; ressaltando que esta classificação setorial se refere à classificação setorial do banco de dados Economática.

Como o objetivo é a verificação das hipóteses $\mathrm{H}_{1}$ e $\mathrm{H}_{2}$ as quais visam verificar a relação entre o valor de mercado da empresa e as variáveis consideradas para medição de inovação (intangíveis e goodwill) e a influência dessas variáveis de inovação sobre o EBITDA, respectivamente, optou-se pelo cálculo da regressão múltipla. 
Justificando a escolha das variáveis dependentes, o valor de mercado exerce o papel de trazer os reflexos dos fundamentos das firmas na percepção dos acionistas. $O$ EBITDA por sua vez, elimina os efeitos dos financiamentos, e através da comparação deste valor é possível obter um bom indicador da produtividade e eficiência do negócio, além de estar relacionado ao resultado obtido pela firma, já que tem o lucro como base inicial.

Assim o modelo o modelo econométrico utilizado para análise dos dados foi do tipo:

$Y=\alpha+\beta_{1} X_{1}+\cdots+\beta_{n} X_{n}+\varepsilon$

em que:

$Y=$ variáveis dependentes

$X_{1} e X_{n=}$ variáveis independentes

$\varepsilon=$ erro aleatório.

$\alpha=$ intercepto

E, para verificação das hipóteses estabelecidas, definiu-se para $\mathrm{H}_{1}$, o valor de mercado como variável dependente e para $\mathrm{H}_{2}$ o EBITDA; para ambas as hipóteses, as variáveis: intangível e goodwill foram determinadas como independentes, conforme consta no Quadro 1 a seguir.

\begin{tabular}{|l|l|l|}
\hline Hipóteses & Variáveis dependentes & Variáveis independentes \\
\hline $\mathbf{H}_{\mathbf{1}}$ & Valor de mercado & Intangível e goodwill \\
\hline $\mathbf{H}_{2}$ & EBITDA & Intangível e goodwill \\
\hline
\end{tabular}

Quadro 1 - Variáveis do modelo

Fonte: Dados da pesquisa 
Cabe ressaltar que para fins de ajustamento do modelo, optou-se por utilizar os logaritmos neperianos das variáveis selecionadas. Após a preparação das variáveis no software Excel, fez-se os cálculos da regressão múltipla utilizando o software SPSS Statistical Package for the Social Science, versão 20.0.

A opção pela utilização da regressão múltipla se deu por esta ser uma técnica estatística que permite avaliar a relação entre uma variável dependente e muitas variáveis independentes (BISQUERRA et al., 2004; DANCEY; REIDEY, 2006). Hair Jr. et al. (2009) afirmam que se deve utilizar a regressão múltipla quando se possui os valores das variáveis independentes e se deseja prever os valores das variáveis dependentes, ou seja, trata-se de uma técnica de dependência que pode fornecer previsão e explicação dos eventos que estão sendo estudados.

\section{DISCUSSÃO DOS RESULTADOS}

Os testes estatísticos foram realizados em separado, objetivando testar a $\mathrm{H}_{1}$ e $\mathrm{H}_{2}$.

Primeiramente foram analisados os dados relacionados à $\mathrm{H}_{1}$, isto é, se o valor de mercado das cinco firmas listadas é impactado pelo indicador de inovação, neste estudo determinado como composto pelo intangível e goodwill. Os resultados encontrados seguem na Tabela 1.

Tabela 1 - Verificação de $\mathrm{H}_{1}$

\begin{tabular}{l|l|l|l|l}
\hline Hipótese & $\mathbf{R}$ & $\mathbf{R 2}$ & $\mathbf{R 2}$ ajustado & Significância \\
\hline $\mathbf{1}$ &, $704^{\mathrm{a}}$ &, 495 &, 458 & $*$ \\
\hline
\end{tabular}

${ }^{*}$ Significativo a 0,01

Fonte: Dados da pesquisa. 
Analisando o coeficiente de correlação $R^{2}$ que mede a proporção da variação que é explicada pela variável independente no modelo de regressão, verifica-se que as variáveis escolhidas explicam em quase $50 \%$ a influência da inovação no valor de mercado das empresas. Assim, a relação linear entre o valor de mercado e o indicador de inovação é considerada relevante, pois, o modelo é significativo, ou seja, é possível afirmar, nesta amostra, que os valores alocados em intangíveis e em goodwill são refletidos no valor de mercado das empresas, explicando, em parte, seu crescimento. Provavelmente, a ligação destas variáveis independentes ao valor de mercado se deva à valorização das marcas, amplamente conhecidas, e que acabam por compor uma parcela de todas as variáveis envolvidas. Assim, pode-se atestar que a hipótese $\mathrm{H}_{1}$ pode ser confirmada para a amostra deste estudo, ressaltando que devam existir outras variáveis que impactam o valor de mercado das empresas.

Procedendo-se à análise de $\mathrm{H}_{2}$, ou seja, se os EBITDA's das empresas da amostra são impactados pelos valores destinados aos intangíveis e ao goodwill, têm-se os resultados explicitados na Tabela 2, a seguir.

Tabela 2 - Verificação de $\mathrm{H}_{2}$

\begin{tabular}{l|l|l|l|l}
\hline Hipótese & $\mathbf{R}$ & $\mathbf{R}^{2}$ & $\mathbf{R}^{2}$ ajustado & Significância \\
\hline $\mathbf{2}$ &, $743^{\text {a }}$ &, 552 &, 518 & $*$ \\
\hline
\end{tabular}

*Significativo a 0,01

Fonte: Dados da pesquisa.

Verificando o coeficiente de correlação $R^{2}$, constata-se que, neste caso, 0 modelo dois apresentou comportamento semelhante ao visto no modelo 1 , com resultados levemente superior. Assim, pode-se concluir que a inovação também impacta na variável EBITDA. O modelo explica em mais de $50 \%$ a influência dessas variáveis.

É possível afirmar, nesta amostra, que os valores alocados em intangíveis e em goodwill são refletidos no EBITDA, explicando, em parte, seu crescimento. Tal valor se 
deve provavelmente à reconhecida capacidade do EBITDA como indicador da tendência de lucros na atividade principal da empresa, tendo em vista alguns autores relacionarem o goodwill como um lucro além do esperado. Assim, pode-se atestar que a hipótese $\mathrm{H}_{2}$ pode ser confirmada para a amostra deste estudo, ressaltando que devam existir outras variáveis que impactam o valor de mercado das empresas.

Insta salientar que os coeficientes analisados foram os já ajustados pelo software, em ambos os casos. Optou-se por utilizar os coeficientes já ajustados por questões de conservadorismo estatístico.

\section{CONSIDERAÇÕES FINAIS}

Com a realização dos testes estatísticos foi investigada a validade de duas hipóteses que relacionam o valor de mercado das empresas e o EBITDA a um indicador de inovação, composto pelas variáveis intangíveis e goodwill, a fim de identificar a existência de relação entre os indicadores financeiros e ganhos financeiros para as empresas, decorrentes de seus esforços de inovação.

Os resultados empíricos geraram evidências de que o valor de mercado sofre influências dos esforços inovadores das empresas o que corrobora a ideia de que quanto maior a firma mais pujante é sua capacidade de inovar, pois possui possibilidade de investir no desenvolvimento de inovações de forma mais robusta. Ademais, as grandes corporações possuem envergadura para possuírem setores destinados, especificamente, para o desenvolvimento de inovações.

No que tange ao relacionamento entre a competência de as empresas da amostra terem sua capacidade de geração lucro, representada aqui pelo EBITDA, influenciada por investimentos em inovação, ficou evidente que inovar torna a empresa mais rentável e, consequentemente, irá gerar valor para o acionista.

Destarte, ambas as hipóteses propostas podem ser comprovadas, sendo que a inovação apresenta impacto pouco superior na variável EBITDA. A confirmação de $\mathrm{H}_{1} \mathrm{e}$ $\mathrm{H}_{2}$ responde, em parte, ao objetivo geral deste estudo, haja vista a influência da 
inovação no valor de mercado e no EBITDA das empresas. Entretanto, os modelos apresentaram um $\mathrm{R}^{2}$ mediano, ou seja, há outras variáveis que podem compor 0 modelo econométrico, a fim de que se consiga um poder de explicação maior com os mesmos. Assim, não foi possível verificar se as empresas de maiores retornos financeiros são as que mais inovam.

Os resultados encontrados não são suficientes para responder se os valores despendidos em inovação consistem em investimentos, isto é, geram retornos para a firma ou se constituem gastos. Faz-se necessária a realização de estudos mais aprofundados, com amostra representativa e considerando um intervalo de tempo mais expressivo. Insta salientar que este estudo possui como restrições o fato de o espaço temporal ser relativamente pequeno, devido à obrigatoriedade do intangível e goodwill serem recentes nos demonstrativos contábeis das empresas de capital aberto no Brasil.

Sugere-se para estudos futuros, ampliar a discussão e o estabelecimento de estudos empíricos que proponham indicadores capazes de mensurar, de forma sistemática, a influência da inovação sobre os retornos das firmas.

\section{REFERÊNCIAS}

ANTUNES, M.T.P.; LEITE, R. S. (2008). Divulgação de informações sobre ativos intangíveis e sua utilidade para analistas de investimentos. Revista Universo Contábil, v. 4, n. 4, p. 22-38.

ASSAF NETO, A. (2002). Estrutura e análise de balanço. São Paulo: Atlas.

BISQUERRA, R.; SARRIERA, J.C.; MARTINEZ, F. (2004). Introdução à Estatística: Enfoque Informático com o Pacote Estatístico SPSS. Porto Alegre: Artmed.

CARVALHO JUNIOR, N. S.; RUIZ, R.M. (2008). Determinantes do desempenho das firmas a partir das novas capacitações internas: um estudo de firmas brasileiras. Rio de Janeiro: Revista Economia Contemporânea, v. 12, n. 1, p.97-127, jan,/abr.

COLAUTO, R. D.; NASCIMENTO, P.S.; AVELINO, B.C.; OSCAR NETO, A.B. (2009). Evidenciação de ativos intangíveis não adquiridos nos relatórios da administração das 
Inovação: Gasto ou Investimento?

Déborah Mara Siade Barbosa, Nayara Silva de Noronha, Thaís Alves dos Santos, Mário Sérgio de

Almeida

companhias listadas nos níveis de governança corporativa da Bovespa. Contabilidade Vista \& Revista, v. 20, n. 1, p. 142-169, jan./mar.

CRISÓSTOMO, V. (2009). Ativos intangíveis: estudo comparativo dos critérios de reconhecimento, mensuração e evidenciação adotados no Brasil e em outros países. Contabilidade, Gestão e Governança, v. 12, n. 1, p. 50-68.

CVM - Comissão de Valores Mobiliários. (2007). Instrução CVM n.457 de 13 de julho de 2007. Dispõe sobre a elaboração e divulgação das demonstrações financeiras consolidadas, com base no padrão contábil internacional emitido pelo International Accounting Standardas Board.

DANCEY, C. P.; REIDY, J. (2006). Estatística sem matemática para psicologia: usando SPSS para Windows. (3 ed.). Porto Alegre: Artmed.

DARROCH, J.; McNAUGHTON, R. (2002). Examining the link between knowledge management practices and types of innovation. Journal of Intellectual Capital, v. 3, n. 3, p. $210-222$.

EDVINSSON, L.; DVIR, R.; NORTH, N. (2004). Innovations: the new unit of analysis in the knowledge era: the quest and context for innovation efficiency and management of IC. Journal of Intellectual Capital, v. 5, n. 1, p. 40-58.

GIL, A. C. (2002). Como elaborar projetos de pesquisa. (4 ed.). São Paulo: Atlas.

HAIR, J. F., ANDERSON, R. E., TATHAM, R. L. BLACK, W. C. Análise multivariada de dados. Porto Alegre: Artmed, 2009.

HENDRICKSEN, E. S.; VAN BREDA, M.F. (1999). Teoria da contabilidade. Tradução de Antonio Zoratto Sanvicente. (5 ed.). São Paulo: Atlas.

IUDíCIBUS, S. de. (2004). Teoria da contabilidade. (7 ed.). São Paulo: Atlas.

KUPFER, D.; HASENCLEVER, L. (2002). Economia industrial: fundamentos teóricos e práticas no Brasil. Rio de Janeiro: Campus.

LEV, B. (2001). Intangibles: management, measurement and reporting. Washington: Brookings Institution Press.

MARION, J.C. Reflexões sobre Ativo Intangível. Disponível em: http://www.marion.pro.br/portal/modules/wfdownloads/visit.php?cid=2\&lid=7 Acesso: 04/dez/2011. 
Inovação: Gasto ou Investimento?

Déborah Mara Siade Barbosa, Nayara Silva de Noronha, Thaís Alves dos Santos, Mário Sérgio de

Almeida

MATESCO, V.R. (1993). Inovação tecnológica das empresas brasileiras: a diferenciação competitiva e a motivação para inovar. (Tese de Doutorado). Rio de Janeiro, IEI/UFRJ.

MEDEIROS, J. B. (2008). Redação científica: a prática de fichamentos, resumos, resenhas. São Paulo: Atlas.

MOREIRA, M. M. (1989). Progresso técnico e estrutura de mercado: o caso da indústria de telequipamentos. Rio de Janeiro: BNDES.

MUDAMBI, R. (2008). Location, control and innovation in knowledge-intensive industries. Journal of Economic Geography, n. 8, p. 699-725.

NARVEKAR, R. S.; JAIN, K. (2006). A new framework to understand the technological innovation process. Journal of Intellectual Capital, v. 7, n. 2, p. 174-186.

OECD - Organization for Economic Co-operation and Development. (2005). Oslo manual: proposed guidelines for collecting and interpreting technological innovation data. Paris: OECD.

PENROSE, E. (1958). A teoria do crescimento da firma. Campinas: Unicamp.

PEREZ, M. M.; FAMÁ, R. (2006). Ativos intangíveis e o desempenho empresarial. Revista Contabilidade \& Finanças, n. 40, p. 7-24, jan./abr.

RICHARDSON, R.J. (1989). Pesquisa social: métodos e técnicas. São Paulo: Atlas.

RIOS, J.A.D.; PINTO, J. S. (2004). A inovação nas empresas e seu processo de mensuração. In: Simpósio de Excelência em Gestão e Tecnologia, Rezende. Anais... Rezende, SEGET. Disponível em: http://www.aedb.br/seget/artigos2004.php. Acesso em: 04/dez/2011.

ROGERS, M. (1998). The definition and measurement of innovation. Melbourne Institute Working Paper, n. 10/98. May.

SCHUMPETER, J.A. (1982). Teoria do desenvolvimento econômico: uma investigação sobre lucros, capital, crédito, juro e o ciclo econômico. São Paulo: Abril Cultural. (Os Economistas).

SHIKIDA, P. F.; BACHA, C. J. (1998). Modernização da Agroindústria Canavieira no Brasil e as Estratégias Tecnológicas das Firmas. Revista Brasileira de Economia. v. 53, n. 1, Jan./Mar. p. 235-254 
TEH, C. C.; KAYO, E. K.; KIMURA, H. (2008). Marcas, patentes e criação de valor. Revista de Administração Mackenzie, v. 9, n. 1, p. 86-106.

TIRONI, L. F.; CRUZ, B. O. (2008). Inovação incremental ou radical: há motivos para diferenciar? Uma abordagem com dados da PINTEC. Texto para Discussão $n^{\circ} 1360$. Rio de Janeiro: Ipea, out.

VASCONCELOS, Y. L. (2002). EBITDA como instrumento de avaliação de empresas. Revista Brasileira de Contabilidade. Brasília, n.136, p.38-47, jul./ago.

VERGARA, S. C. (2006). Métodos de Pesquisa em Administração. (2 ed.). São Paulo: Atlas, 287p.

Data de Submissão: 06/12/2012

Data de Aceite: 28/12/2012 